\title{
Dynamical elastic contact of a rope with the ground
}

\author{
Gregory Kozyreff $\odot^{*}$ and Benoît Seron $\odot$ \\ Optique Nonlinéaire Théorique, Université libre de Bruxelles (U.L.B.), CP 231, Campus de la Plaine, 1050 Bruxelles, Belgium
}

(Received 6 October 2020; revised 20 January 2021; accepted 6 May 2021; published 21 June 2021)

\begin{abstract}
A rope laid on the ground with one end subjected to time-dependent forcing is proposed as a prototypical elastic dynamical contact problem, which we study analytically, numerically, and experimentally. The dynamics is governed by an infinite set of linear and nonlinear resonances. In the limit of weak bending stiffness, the fundamental frequency is found to be independent of the rope tension. A transition between a radiation-less and a wave radiating state occurs via a series of grazing bifurcations, whereby new contacts between the rope and the ground are formed. The grazing bifurcations form overlapping Arnold tongues in the frequency-amplitude parameter space. Finally, for ropes with large bending stiffness and when the geometric nonlinearity is important, bistability is observed between several wave-making regimes.
\end{abstract}

DOI: 10.1103/PhysRevResearch.3.L022026

Take a long piece of rope, lay it on the ground, and give it a sudden vertical shake at one extremity. If vigorous enough, you are likely to see a bell-shape elevation traveling far away along the rope, until internal friction makes it disappear. Such a wave is familiar to anyone and simple looking, but the underlying physics is more complicated than meets the eye. In this Letter, we shed some light on the dynamics underlying wave generation by a combination of analytical, numerical, and experimental results. Aside from its recreational aspect, this problem is an opportunity to gather basic qualitative and quantitative knowledge about the theory of dynamical contacts involving deformable bodies. Indeed, questions of this kind can usually be approached only numerically [1,2]. Even in the static case, analytically tractable elastic contact problems are rare and most often proceed from Hertz's famous study of two spheres pressed against one another [3]. On the other hand, the present problem has a practical interest, as it may be connected to some aspects of cable laying on ocean beds $[4,5]$, rails deformation under a moving load [6], the intrusion of a rod into a cylinder in the context of oil well drilling [7], or parasitic contacts in rotating machines [8]. Importantly, and contrary to other systems combining vibration and impact $[9,10]$, as in an atomic force microscope in the tapping regime [11], the contact point is not known in advance and becomes nonunique on the occasion of wave emission.

To study wave generation in a systematic way, we set up an experiment, schematically depicted in Fig. 1, where a long rope is excited harmonically with a small amplitude. Intuitively, one would expect that below a certain threshold frequency, the lifted part of the rope does no more than

\footnotetext{
*gregory.kozyreff@ulb.be
}

Published by the American Physical Society under the terms of the Creative Commons Attribution 4.0 International license. Further distribution of this work must maintain attribution to the author(s) and the published article's title, journal citation, and DOI. gently follow the motion imparted at the extremity and that, above, waves are radiated along the rope. We determine such a frequency and find, unexpectedly, that it is independent of the rope tension when bending stiffness is negligible. Further analysis reveals that wave generation starts at grazing bifurcations. Next, we show that (i) the wave-less state can regain stability by increasing the driving frequency above the fundamental frequency and (ii) waves can also be generated below this frequency through nonlinear amplification. As a result, the stability boundary of the waveless state displays a set of minima in the amplitude vs frequency parameter space, which are called Arnold tongues in dynamical systems theory. Finally, we experimentally observe hysteresis between a regime of large-wave emission and another one where only small waves are radiated; here too, the boundary of bistability indicates an Arnold tongue pattern.

In the experiment, one end of the rope, at $x=0$, is initially lifted to a height $Z_{0}$ and then made to oscillate vertically and harmonically around that position. The elevation $w(x, t)$ of the rope that is not in contact with the ground obeys the beam equation [12]

$$
\rho \frac{\partial^{2} w}{\partial t^{2}}=T \frac{\partial^{2} w}{\partial x^{2}}-B \frac{\partial^{4} w}{\partial x^{4}}-\rho g,
$$

where $\rho$ is the line density, $B$ is the bending stiffness, and $g$ is the acceleration due to gravity. We assume that the rope is under tension $T$. This tension could naturally arise from static friction with the ground, as one lifts one extremity of the rope, or it could be directly applied, as in our experimental setup. Before shaking the rope, we manually apply a gentle tension on the rope, on the order of $1 \mathrm{~N}$ (as was measured in a static configuration with a force meter), and maintain the resulting static deformation by placing a weight at the unlifted end of the rope (see Fig. 1). This is done to maintain a small slope, which is required for the validity of Eq. (1). In practice, due to space constraints, the rope made an angle of approximately $30^{\circ}$ with the horizontal at the actuated end. In what follows, we assume for simplicity that $T$ is kept constant 


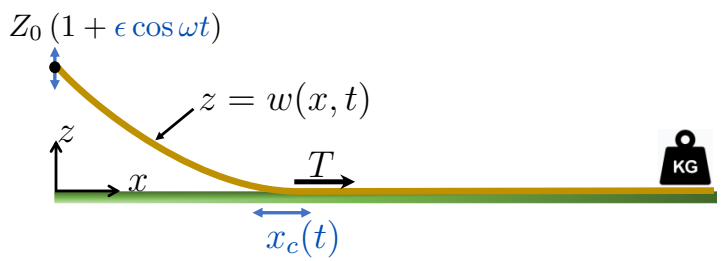

FIG. 1. Schematic of the rope with one end vibrated with relative amplitude $\epsilon$ and pulsation $\omega$ around a fixed elevation $Z_{0}$. Prior to vibrating the rope, a tension $T$ is applied and the resulting static deformation is maintained by a weight placed at the other end of the rope. The rope is sufficiently long to neglect reflection from the weight. For $\epsilon$ sufficiently small, only one coordinate $x_{c}(t)$ describes the edge of the contact region between the rope and the ground (contact point).

and uniform in $x$, which neglects variations associated to stretching or slipping along the ground and requires the slope to remain small [13]. This is in contrast with recent studies on the formation of rucks $[14,15]$ where that force is deduced through the constraint of inextensibility. Note that rucks are essentially under compression rather than in tension, leading to a completely different dynamics. At $x=0$, one has

$$
w=Z_{0}+\epsilon \Delta Z(t), \quad \Delta Z(t) / Z_{0}=\cos \omega t, \quad \epsilon \ll 1,
$$

and, in the absence of an applied moment, $\frac{\partial^{2} w}{\partial x^{2}}=0$. At the contact point $x=x_{c}(t)$, the boundary conditions are $w=$ $\frac{\partial w}{\partial x}=\frac{\partial^{2} w}{\partial x^{2}}=0$. Our aim is to describe the rope dynamics as a function of $\epsilon$ and $\omega$.

In spite of appearances, the above differential problem is strongly nonlinear: The solution $w(x, t)$ of Eq. (1) depends nonlinearly on $x_{c}(t)$, which, in turn, is a functional of $w(x, t)$. A hint of nonlinear behavior can already be found from traveling-wave solutions of Eq. (1) far down the rope in that their speed depends on their amplitude [12]. Before embarking on the analysis, we note that a considerable simplification of Eq. (1) can be made. In our experiments, we use stranded wires. We estimate their bending rigidity by measuring the torque required to make them conform to a quarter circle of prescribed radius. For the thickest wire (diameter $6 \mathrm{~mm}$ ), we find $B \approx 0.01 \mathrm{~N} \mathrm{~m}^{2}$. Hence, over a length scale of $1 \mathrm{~m}$ and with $T \approx 1 \mathrm{~N}$, one clearly has $B \frac{\partial^{4} w}{\partial x^{4}} \ll T \frac{\partial^{2} w}{\partial x^{2}}$ and the bending term can safely be neglected everywhere except in boundary layers near $x=0$ and $x=x_{c}$. Therefore, even though we numerically simulate Eq. (1) we focus, for the sake of analytical investigation, on

$$
\frac{\partial^{2} w}{\partial t^{2}}=c^{2} \frac{\partial^{2} w}{\partial x^{2}}-g, \quad c^{2}=T / \rho
$$

subjected to Eq. (2) and only $w=\frac{\partial w}{\partial x}=0$ at $x=x_{c}$. Expanding $x_{c}$ as

$$
x_{c} \sim x_{0}+\epsilon x_{1}+\epsilon^{2} x_{2}+\cdots,
$$

the boundary conditions can be expanded as

$$
Y\left(x_{0}, t\right)+\left.\left(\epsilon x_{1}+\epsilon^{2} x_{2}\right) \frac{\partial Y}{\partial x}\right|_{x_{0}}+\left.\epsilon^{2} \frac{x_{1}^{2}}{2} \frac{\partial^{2} Y}{\partial x^{2}}\right|_{x_{0}}+\cdots=0,
$$

where $Y=w, \frac{\partial w}{\partial x}$. Furthermore, $w$ is also expanded as

$$
w \sim w_{0}+\epsilon w_{1}+\epsilon^{2} w_{2}+\cdots .
$$

Substituting the above expansions into Eq. (3), the leadingorder problem is a static one and is easily solved:

$$
w_{0}=\frac{g\left(x-x_{0}\right)^{2}}{2 c^{2}}, \quad x_{0}^{2}=\frac{2 c^{2} Z_{0}}{g}=\frac{2 T Z_{0}}{\rho g} .
$$

At the next order, we have $\left(\frac{\partial^{2}}{\partial t^{2}}-c^{2} \frac{\partial^{2}}{\partial x^{2}}\right) w_{1}=0$, which has the general solution $w_{1}=F(t-x / c)+G(t+x / c)$. Using Eq. (2), one has $F(t)+G(t)=\Delta Z(t)$. Next, the two boundary conditions (5) yields $F\left(t-x_{0} / c\right)+G\left(t+x_{0} / c\right)=$ 0 and $-F^{\prime}\left(t-x_{0} / c\right)+G^{\prime}\left(t+x_{0} / c\right)=-g x_{1}(t) / c$. Eliminating the functions $F$ and $G$ from these three equations, we find

$$
x_{1}(t)-x_{1}\left(t-2 x_{0} / c\right)=(2 c / g) \Delta Z^{\prime}\left(t-x_{0} / c\right) .
$$

The above equation describes the linear response of the contact point to a general small-amplitude excitation $\epsilon \Delta Z(t)$. In the particular case of a harmonic excitation $\Delta Z / Z_{0}=\cos \omega t$, one easily finds, using Eq. (7), that

$$
x_{1}=\frac{x_{0} \cos \omega t}{2 \operatorname{sinc}\left(\omega x_{0} / c\right)},
$$

where $\operatorname{sinc}(x)=\sin (x) / x$. An infinite linear response is thus found at frequencies $\omega /(2 \pi)=f_{p}$ where

$$
f_{p}=p \sqrt{\frac{g}{8 Z_{0}}}, \quad p=1,2, \ldots
$$

The set of these resonances make the rope equivalent to a resonator of length $x_{0}$, in which waves can travel at speed $c$. What is surprising, however, is that the tension $T$ is absent from the expression of the resonances. This is because both $c$ and $x_{0}$ increase in proportion to $\sqrt{T}$. The fundamental resonance is akin to that of a classical pendulum of length $2 Z_{0} / \pi^{2}$. Alternatively, Eq. (1) can be recast in dimensionless form to show that $T$ disappears from the mathematical formulation in the limit $B /\left(T x_{0}^{2}\right) \rightarrow 0$. Indeed, with $\xi=x / x_{0}, W=w / Z_{0}$ and $\tau=c t / x_{0}$, Eq. (1) becomes

$$
\frac{\partial^{2} W}{\partial \tau^{2}}=\frac{\partial^{2} W}{\partial \xi^{2}}-2-\beta \frac{\partial^{4} W}{\partial \xi^{4}}, \quad \beta=\frac{B}{T x_{0}^{2}}=\frac{\rho g B}{2 T^{2} Z_{0}},
$$

with $W-1-\epsilon \cos \Omega \tau=W_{\xi}=0$ at $\xi=0$ and $W=W_{\xi}=$ $W_{\xi \xi}=0$ at $\xi=\xi_{c}(\tau)$, and

$$
\Omega=\omega x_{0} / c=\omega \sqrt{2 Z_{0} / g} .
$$

In the limit $\beta \rightarrow 0$, the tension is thus scaled out of the problem. For nonzero $\beta$, the frequencies $f_{p}$ are not equispaced anymore and $f_{1}$ increases slightly, up to $42 \%$ as $\beta \rightarrow \infty[16]$.

We have checked the independence of $f_{1}$ on $T$ experimentally. We mounted a stepper motor (Nema 23) capable of delivering a torque of up to $3 \mathrm{~N} \mathrm{~m}$. The motor was driven by a QGL-HQ MA860H pilot, whose signal came from an Arduino Mega2560. The rotation of the motor was converted into vertical motion by using a home-made Scott Russel linkage. With this setup, a precise motion $\Delta Z(t)$ could be imparted on 
TABLE I. Experimental demonstration of independence of resonance frequency on the tension in the rope. $\rho=15 \mathrm{~g} / \mathrm{m}, B \approx 0.0005$ $\mathrm{N} \mathrm{m}^{2}, \epsilon=0.05, Z_{0}=81 \mathrm{~cm}, \sqrt{g / 8 Z_{0}} \approx 1.23 \mathrm{~Hz}$.

\begin{tabular}{llllllll}
\hline \hline$x_{0} \pm 0.03(\mathrm{~m})$ & 1.08 & 1.20 & 1.47 & 1.65 & 1.80 & 2.30 & 2.45 \\
$f_{1} \pm 0.02(\mathrm{~Hz})$ & 1.18 & 1.19 & 1.20 & 1.20 & 1.23 & 1.17 & 1.20 \\
\hline \hline
\end{tabular}

the rope. We used a thin stranded metallic wire rope (diameter $2 \mathrm{~mm}$ ) with $\rho=15 \mathrm{~g} / \mathrm{m}, B \approx 0.0005 \mathrm{~N} \mathrm{~m}^{2}$. The static elevation $Z_{0}$ was $0.81 \mathrm{~m}$, yielding a theoretical fundamental resonance $\sqrt{g / 8 Z_{0}} \approx 1.23 \mathrm{~Hz}$. Note that the slope of the rope is not very small at $x=0$. Nevertheless, the rope rapidly becomes horizontal as it nears the ground. Additionally, we found that the static profile was almost undistinguishable from a quadratic one, as in Eq. (7), away from the immediate vicinity of the origin. Equations (1) and (3) thus appear to be reliable. To avoid sideways motion of the rope, also known as "ponytail instability" $[17,18]$, the rope was vertically oscillated against a board. This produced some friction, but much less than the internal one. According to the linear theory, a general driving $\Delta Z(t) / Z_{0}=\int A(\omega) \exp (i \omega t) d \omega$ yields a linear response

$$
x_{1}(t)=\frac{x_{0}}{2} \int \frac{A(\omega) e^{i \omega t}}{\operatorname{sinc}\left(\omega x_{0} / c\right)} d \omega .
$$

Hence, in order to excite the fundamental resonance only, $\Delta Z(t)$ was gradually ramped from a vanishing amplitude to a constant sinusoidal excitation in 20 periods of oscillation. In this way, $A(\omega)$ was strongly peaked around a well-defined frequency and unwanted excitation of high-order poles in Eq. (13) was reduced. We increased the frequency until resonance was detected, in the form of a transition to small waves being emitted down the rope. Because of the very small amplitude of the waves, there was some uncertainty on the frequency at the transition on the order of $0.02 \mathrm{~Hz}$. We repeated the experiment with different values of the tension $T$, measured by the static part $x_{0}$ of the contact point. To measure $T$ directly was difficult to implement but, from Eq. (7), $x_{0}$ increases monotonically with $T$. Independence of $f_{1}$ on $x_{0}$ thus implies independence of $T$, all other parameters being unchanged. The results, shown in Table I, confirm the prediction.

Equation (9) indicates that, starting from a small frequency below $f_{1}$ and increasing it, the response of the system diverges. One can anticipate that the unbounded growth of the oscillation can only resolve itself into a train of waves along the rope. On the other hand, in the range $f_{1}<\omega /(2 \pi)<f_{2}$, the linear response becomes small again, suggesting that it is possible not to emit waves there. However, apart from the divergence, the above linear theory fails to give details on how waves are actually generated. Proceeding to higher orders of the analysis, we use the coordinate $s=\left[x-x_{c}(t)\right] / x_{0}$ and the reduced pulsation $\Omega=\omega x_{0} / c$. For a purely harmonic forcing, we find [16] that

$$
w / Z_{0} \sim \sum_{j \geqslant 0}\left(\frac{\epsilon}{\sin \Omega}\right)^{j} F_{j}(s, \epsilon) \cos (j \omega t),
$$

where $F_{0} \sim s^{2}+O\left(\epsilon^{2}\right)$,

$$
\begin{aligned}
F_{1} \sim & (\Omega s-\sin \Omega s)\left[1+\frac{\epsilon^{2} \Omega^{4} \cos ^{2} \Omega}{32 \sin ^{4} \Omega}\left(1+2 \frac{\tan \Omega}{\Omega}\right)\right] \\
& +\frac{\epsilon^{2} \Omega^{4} \sec \Omega}{16 \sin ^{3} \Omega}[\sin 2 \Omega-\sin (2 \Omega+\Omega s)] \sin \Omega s+O\left(\epsilon^{4}\right),
\end{aligned}
$$

$$
\begin{aligned}
F_{2} \sim & \frac{\Omega^{2}}{8}\left[(1-\cos \Omega s)^{2}-\sin ^{2} \Omega s\right. \\
& \left.-\frac{\cos 2 \Omega}{\sin 2 \Omega}(2 \Omega s-\sin 2 \Omega s)\right]+O\left(\epsilon^{2}\right),
\end{aligned}
$$

and

$$
\begin{aligned}
F_{3} \sim & \frac{\Omega^{4}}{32}[\sin \Omega s+\sin 3 \Omega s-2 \sin 2 \Omega s \\
& +\frac{(2-\cos 2 \Omega+\cos 4 \Omega)}{2(1+2 \cos 2 \Omega) \sin ^{2} \Omega}(3 \Omega s-\sin 3 \Omega s) \\
& \left.-4 \frac{\cos 2 \Omega}{\sin 2 \Omega}(1-2 \cos \Omega s) \sin ^{2} \Omega s\right]+O\left(\epsilon^{2}\right) .
\end{aligned}
$$

The leading-order expressions of $F_{2}$ and $F_{3}$ indicate new resonances when $\sin 2 \Omega=0$ and when $1+2 \cos 2 \Omega=0$; that is, when $\omega /(2 \pi)=f_{p} / 2$ or $f_{p} / 3$. Indeed, the nonlinearity in Eq. (5) leads to higher harmonics of the cos $\omega t$ forcing which, in turn, can match the fundamental resonances. As one progresses to higher orders in the analysis, more harmonics are found. In particular, the condition $q \omega /(2 \pi)=f_{p}$ yields the nonlinear resonances $\omega_{p, q} /(2 \pi)=f_{p} / q=(p / q) f_{1}$, similarly to Ref. [8]. Since $\left\{\omega_{p, q} \mid p, q \in \mathbb{Z}\right\}$ is a dense set, any frequency is arbitrarily close to a nonlinear resonance and any harmonic forcing should in principle lead to a divergent response in the absence of dissipation.

Let us study the limit $\omega /(2 \pi) \rightarrow f_{p}$ of the above expansion. Writing $\Omega=p \pi+\mu, \mu \ll 1$, Eqs. (14)-(17) yield, for sufficiently small $\mu$,

$$
\begin{aligned}
w / Z_{0} \sim & s^{2}+(-)^{p} \frac{\epsilon^{3}(p \pi)^{4}}{32 \mu^{5}}[\cos (\omega t)(p \pi s-\sin p \pi s) \\
& \left.+\frac{1}{3} \cos (3 \omega t)(3 p \pi s-\sin 3 p \pi s)\right] .
\end{aligned}
$$

This function displays a local minimum at times $t$ given by $\omega t=(2 n+p) \pi$. One finds that, at such times, the rope makes contact with the ground at $s=-1.13 /(p \pi)$ with zero slope and zero velocity if

$$
\epsilon=\epsilon_{G}(\omega) \approx \frac{0.309}{p^{2}} \mu^{5 / 3}=\frac{0.309}{p^{2}}\left(\frac{\omega x_{0}}{c}-p \pi\right)^{5 / 3} .
$$

Importantly, Arnold tongues given by the above formula become flatter as $p$ increases. Hence, the areas below these curves shrink as $1 / p^{2}$. Similarly, using the $O\left(\epsilon^{4}\right)$ expression for $F_{4}(s, \epsilon)$ [16], one may investigate the region $\omega /(2 \pi) \rightarrow$ $f_{1} / 2$, by writing $\Omega=\pi / 2+\mu, \mu \ll 1$. One then obtains that the rope touches the ground before $x=x_{0}$ if, locally,

$$
\epsilon=\epsilon_{G}(\omega) \approx 1.815 \mu^{3 / 4}=1.815\left(\frac{\omega x_{0}}{c}-\frac{\pi}{2}\right)^{3 / 4} .
$$




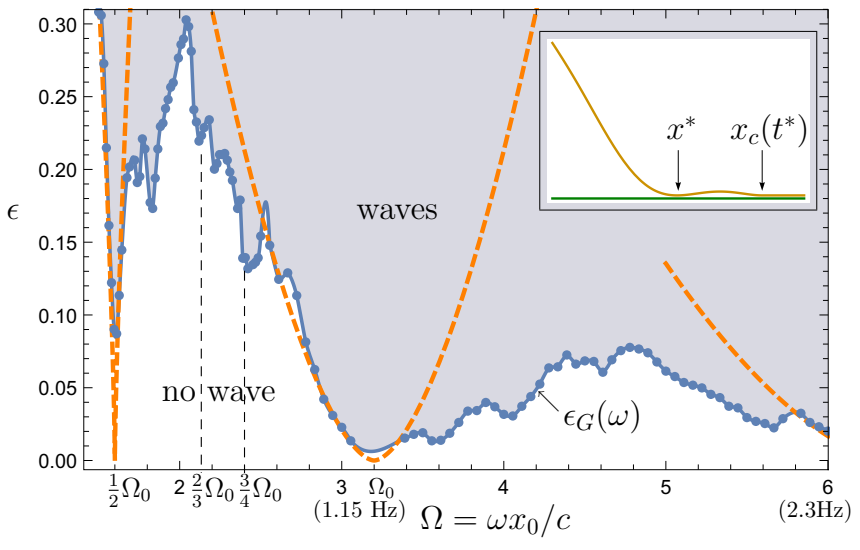

FIG. 2. Bifurcation diagram in the $(\omega, \epsilon)$ plane obtained by simulation of Eq. (1) with $B /\left(T x_{0}^{2}\right)=0.01$. Blue dots: numerics. Orange dashed curves, from left to right: $\epsilon=1.815\left|\Omega-\Omega_{0} / 2\right|^{3 / 4}, \epsilon=$ $0.309\left|\Omega-\Omega_{0}\right|^{5 / 3}$, and $\epsilon=0.077\left|\Omega-2 \Omega_{0}\right|^{5 / 3}$, where $\Omega_{0}=3.2$. Inset shows the analytical profile (18) at impact near the $\Omega_{0}$ resonance. Between parentheses are indicated frequencies in $\mathrm{Hz}$ corresponding to an experiment with $Z_{0}=81 \mathrm{~cm}$.

Equation (18) is not rigorously valid because it breaks the asymptotic ordering of terms in Eq. (14). Therefore, it should not be given any theoretical value other than providing a qualitative trend. Nevertheless, the resulting expression (19) is found to fit remarkably well with the appearance of waves in numerical simulations. Numerical simulations of dynamical contact problems are known to be challenging [2]. A practical issue is the determination and proper processing of the contact set in the discretized rope. We used an algorithm adapted from Liakou et al. [19]. A description is given in the Supplemental Material [16]. Figure 2 shows the boundary between the regimes of no radiation and radiation. Numerically, the fundamental resonance is slightly shifted from $\Omega=\pi$ to $\Omega=\Omega_{0} \approx 3.2$; this results from the small but nonzero bending stiffness $\left[B /\left(T x_{0}^{2}\right)=0.01\right]$. Strikingly, the numerical curve contains a large number of sharp dips, in addition to those predicted by the linear analysis. These are signatures of the nonlinear resonances discussed above. In particular, a sharp resonance is seen at $\Omega_{0} / 2$. As anticipated by the theory, the waveless state regains stability in the range $\Omega_{0}<\omega x_{0} / c<2 \Omega_{0}$ for sufficiently small $\epsilon$. The analytical formulas derived above in the vicinity of $\Omega_{0} / 2, \Omega_{0}$, and $2 \Omega_{0}$ convincingly fit the numerical curve.

The situation described by Eqs. (18) and (19) is reminiscent of a grazing bifurcation. Such a bifurcation classically applies to a point mass attached to a spring and subjected to a periodic force. Upon increasing the forcing amplitude, the mass starts making contact with an obstacle. Beyond the grazing bifurcation, the dynamics rapidly becomes chaotic [20]. The crucial difference here, of course, is that the rope is spatially distributed. Nevertheless, the analogy is sufficiently strong to also call the threshold identified by Eq. (19) a grazing bifurcation. Let us denote by $t^{*}$ and $x^{*}$ the time and location of impact as determined above. If $\epsilon$ exceeds the grazing bifurcation threshold, the contact happens with a finite speed $-V$. In the limit of an infinitely rigid ground with restitution coefficient $r$, the rope locally rebounds instantaneously with speed $r V$. This amounts to a reaction force $(1+r) \rho V \delta(t-$ $\left.t^{*}\right) \delta\left(x-x^{*}\right)$, which produces an elevation $w^{*}(x, t)=0.5(1+$ $r) V x_{0} / c$ in the range $-c\left(t-t^{*}\right)<x-x^{*}<c\left(t-t^{*}\right)$ [12]. The perturbation $w^{*}$ expands in both directions and adds itself to the elevation given by Eq. (14). Ultimately, this provokes the detachment of the bump between $x^{*}$ and $x_{c}\left(t^{*}\right)$ and its propagation at speed $c$ down the rope. In this scenario, the portion of the rope ahead of $x^{*}$ which ultimately forms the traveling wave is initially given by Eq. (18) at $t^{*}$.

We now turn to the experimental demonstration of wave generation through harmonic forcing at $x=0$. As previously mentioned, the computer-driven motor was mounted on a table at a height $Z_{0}=81 \mathrm{~cm}$. The angle of the rotor varied in steps of $1.8^{\circ}$. Given the length of the arm of the Russel linkage, this translates into an uncertainty of $\Delta Z(t)$ of $4 \mathrm{~mm}$, hence an uncertainty $|\Delta \epsilon| \approx 0.005$. The transitions were monitored by varying $\epsilon$ for fixed $\omega$. Being computer controlled, the uncertainty in frequency is estimated to be well under $0.01 \mathrm{~Hz}$ and hence negligible. From what precedes, waves emitted at the grazing bifurcation point $\epsilon_{G}(\omega)$ are of very small amplitude, the maximum being approximately $0.085 Z_{0} /(p \pi)^{2}$ (see inset of Fig. 2). Moreover, past the bifurcation threshold, this amplitude does not grow rapidly as $\left[\epsilon-\epsilon_{G}(\omega)\right]^{1 / 2}$, but, rather, only linearly in $\epsilon-\epsilon_{G}(\omega)$, as in other impact systems [21]. Finally, the waves undergo rapid attenuation due to internal friction between the strands of the rope. This makes the determination of the transition experimentally challenging, and we focused on large frequencies, near $2 \Omega_{0}$, in order to benefit from a large amplification of the oscillations.

Figure 3(a) shows the experimental result. The boundary between no waves and small waves is the grazing bifurcation curve. It shows a minimum near $2.3 \mathrm{~Hz}$, in good agreement with the $f_{2}$ resonance of Fig. 2. However, quantitative agreement between the numerical curve of Fig. 2 and the experimental one in Fig. 3(a) is poor, even though the order of magnitude is the same. We attribute this poor matching to the nonconstancy of $T$. Indeed, $T$ is not actively controlled in the experiment. The fact that the slope of the rope is not everywhere small together with small parasitic slippage on the ground both make $T$ vary in time and space in practice [16].

Next to the small-wave regime described thus far, we experimentally discover a large-wave regime. The two regimes stably coexist over a wide range of parameters. Figures 3(b) and 3(c) are snapshots of the two distinct dynamical states, observed for the same values of $\omega$ and $\epsilon$ [black dot in Fig. 3(a)]. The limit of coexistence between the two regimes is classically given by a curve of limit point $\epsilon_{L P}(\omega)$ in the $(\omega, \epsilon)$ space.

Fortunately, the curve $\epsilon_{L P}(\omega)$ is much more convenient to determine than $\epsilon_{G}(\omega)$ because the transition between the two regimes is visually clear-cut. We record $\epsilon_{L P}(\omega)$ in the following way: (i) start with a value $\epsilon>\epsilon_{L P}(\omega)$ in the state of large-wave emission. (ii) Decrease $\epsilon$ by small steps and wait for the system to relax to a stable operation. (iii) As soon as $\epsilon<\epsilon_{L P}$, the large-wave state irreversibly disappears after only a few oscillations, giving way to the small-wave state. The most important feature of the $\epsilon_{L P}(\omega)$ curve is its minimum. This minimum attests of the resonant nature of that state and 

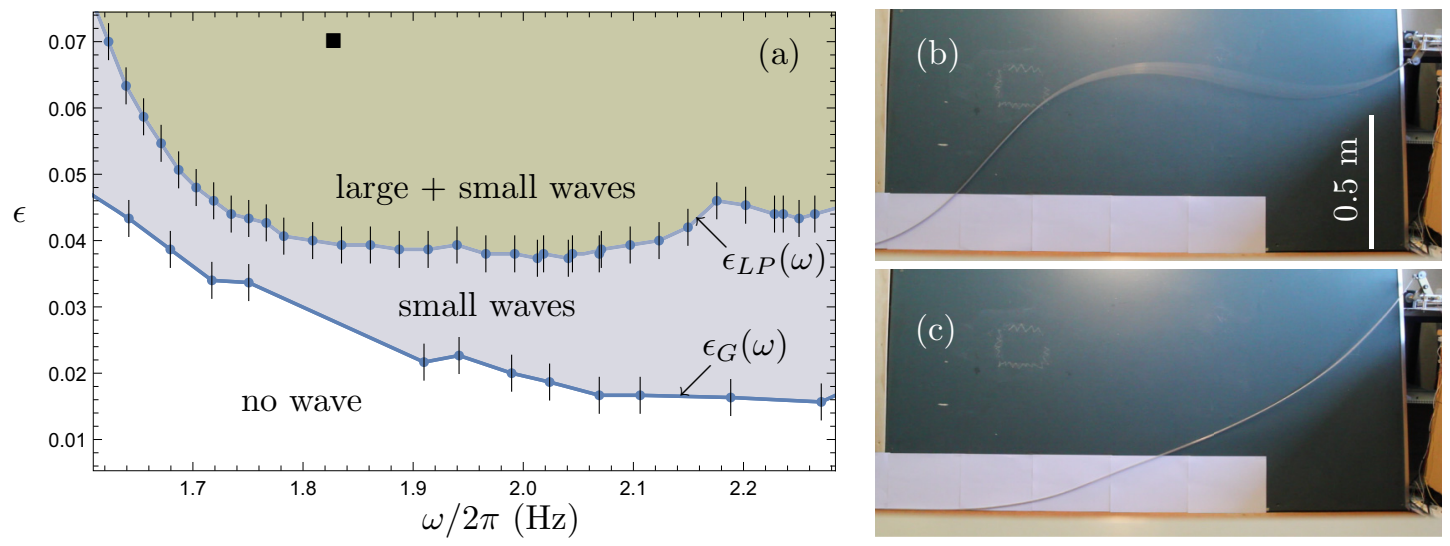

FIG. 3. (a) Experimental bifurcation diagram in the $(\omega, \epsilon)$ parameter plane. $\epsilon_{L P}(\omega)$ : limit-point curve above which large-wave and smallwave regimes coexist. $\epsilon_{G}(\omega)$ : grazing bifurcation curve. (b) Snapshots of the large-wave oscillation. (c) Snapshot of the small-wave oscillation (extracted frames from video in the Supplemental Material [16].) Panels (b) and (c) both correspond to the same value of $\omega$ and $\epsilon$ but different initial conditions [black square in panel (a)]. $\rho=48 \mathrm{~g} / \mathrm{m}, B \approx 0.01 \mathrm{~N} \mathrm{~m}^{2}, Z_{0}=81 \mathrm{~cm}$.

is an indirect manifestation of the Arnold tongues described above.

Regarding the large-wave-emitting state, we make two observations. First, we could not observe it with ropes of smaller cross sections, i.e., smaller bending stiffness $B$. This suggests that bending stiffness plays an important role in the existence of this dynamical regime. Second, we could not numerically reproduce the bistability between a large-waveand a small-wave-making state for any value of $B$ in Eq. (1). From this, we conclude that this model is insufficient to describe this state and that a fully geometrically nonlinear model is required $[12,13,17]$. Geometrical nonlinearity couples the transverse motion $w(x, t)$ to a longitudinal motion, making the displacement fully vectorial. This could be the subject of further investigation.

Dynamical contacts between deformable bodies may display rich dynamical behavior. Using a simple rope as a prototype example, we have seen that the dynamics combines the physics of free moving boundaries and that of nonsmooth dynamical systems. In particular, contacts are governed by the infinite set of resonant deformation modes of the rope [see the multiple poles in Eq. (8)], supplemented by their nonlinear harmonics. When analyzing more complicated dy- namical contact problems, where analytical results are out of reach, the present study suggests to pay attention to linear resonances and their nonlinear harmonics. In the vicinity of these resonances, nonlinear waves and chaos are susceptible to arise via grazing bifurcations. The stability regions are likely to be again delimited by Arnold tongues in the parameter space. Thanks to the slenderness of the rope, the elastic degrees of freedom are reduced to their simplest expression here. Next in complexity would be the study of time-dependent contacts between two slender bodies and the inclusion of out-of-plane degrees of freedom. At the experimental level, it would be interesting to carry out a more complete and systematic study than the present demonstration. It would, for instance, be interesting to directly monitor the tension within the rope and to manufacture ropes with controlled properties. Finally, the effect of adhesion could be investigated.

B.S. is a Research Fellow and G.K. is a Research Associate of the Fonds de la Recherche Scientifique - FNRS (Belgium.) We thank Pascal Damman and Fabian Brau for stimulating discussions and Jamal Tahmaoui for fabricating the Scott Russel linkage.
[1] P. Wriggers, Computational Contact Mechanics (Springer, Berlin, Heidelberg, New York, 2006) .

[2] D. Doyen, A. Ern, and S. Piperno, Time-integration schemes for the finite element dynamic Signorini problem, SIAM J. Sci. Comput. 33, 223 (2011).

[3] H. Hertz, Miscellanous Papers (Macmillan, London, New York, 1896) .

[4] E. E. Zajac, Dynamics and kinematics of the laying and recovery of submarine cables, Bell Syst. Tech. J. 36, 1129 (1957).

[5] M. K. Jawed, F. Da, J. Joo, E. Grinspun, and P. M. Reis, Coiling of elastic rods on rigid substrates, Proc. Natl. Acad. Sci. U.S.A. 111, 14663 (2014).

[6] Y. Weitsman, Onset of separation between a beam and a tensionless elastic foundation under a moving load, Int. J. Mech. Sci. 13, 707 (1971).
[7] J. T. Miller, C. G. Mulcahy, J. Pabon, N. Wicks, and P. M. Reis, Extending the reach of a rod injected into a cylinder through distributed vibration, J. Appl. Mech. 82, 021003 (2015).

[8] Md A. Mokhtar, A. K. Darpe, and K. Gupta, Investigations on bending-torsional vibrations of rotor during rotor-stator rub using lagrange multiplier method, J. Sound Vib. 401, 94 (2017).

[9] V. K. Astashev and V. L. Krupenin, Experimental investigation of vibrations of strings interacting with point obstacles, Dokl. Phys. 46, 522 (2001).

[10] K. D. Murphy and T. M. Morrison, Grazing instabilities and post-bifurcation behavior in an impacting string, J. Acoust. Soc. Am. 111, 884 (2002).

[11] A. J. Dick, B. Balachandran, H. Yabuno, M. Numatsu, K. Hayashi, M. Kuroda, and K. Ashida, Utilizing nonlinear 
phenomena to locate grazing in the constrained motion of a cantilever beam, Nonlinear Dyn. 57, 335 (2009).

[12] P. Howell, G. Kozyreff, and J. Ockendon, Applied Solid Mechanics (Cambridge University Press, Cambridge, 2009).

[13] B. D. Coleman and E. H. Dill, Flexure waves in elastic rods, J. Acoust. Soc. Am. 91, 2663 (1992).

[14] J. M. Kolinski, P. Aussillous, and L. Mahadevan, Shape and Motion of a Ruck in a Rug, Phys. Rev. Lett. 103, 174302 (2009).

[15] D. Vella, A. Boudaoud, and M. Adda-Bedia, Statics and Inertial Dynamics of a Ruck in a Rug, Phys. Rev. Lett. 103, 174301 (2009).

[16] See Supplemental Material at http://link.aps.org/supplemental/ 10.1103/PhysRevResearch.3.L022026 for a discussion of the validity of Eq. (1), the effect of bending stiffness and dissipation on the linear response, details of the weakly nonlinear analysis and the numerical algorithm, and experimental movies.

[17] A. Belmonte, M. J. Shelley, S. T. Eldakar, and C. H. Wiggins, Dynamic Patterns and Self-Knotting of a Driven Hanging Chain, Phys. Rev. Lett. 87, 114301 (2001).

[18] J. B. Keller, Ponytail motion, SIAM J. Appl. Math. 70, 2667 (2010).

[19] A. Liakou, V. Denoël, and E. Detournay, Fast in-plane dynamics of a beam with unilateral constraints, J. Eng. Mech. 143, 04016116 (2017).

[20] S. W. Shaw and P. J. Holmes, A periodically forced piecewise linear oscillator, J. Sound Vib. 90, 129 (1983).

[21] M. di Bernardo, C. J. Budd, A. R. Champneys, P. Kowalczyk, A. B. Nordmark, G. O. Tost, and P. T. Piiroinen, Bifurcations in nonsmooth dynamical systems, SIAM Rev. 50, 629 (2008). 